\title{
Cosine Distance Method
}

National Cancer Institute

\section{Source}

National Cancer Institute. Cosine Distance Method. NCI Thesaurus. Code C72662.

A type of pearson measure, which considers the relative differences (e.g.

$A * B / A b s[A] * A b s[B]$ ) assuming that the scale is uniform (that the distance from zero is relative). In some cases this can give better results, particularly where the data is not 'normally' distributed. 